\title{
Cut-off Value for Stenosis Ratio and Zung Depression Scale in Successful Prediction of Posterior Spinal Fusion surgery
}

\author{
Mahsa Babaee ${ }^{1,2}$, Alireza Zali ${ }^{*}$, Mohammad Mehdi Sepehri ${ }^{3}$, Zahra Razzaghi ${ }^{4}$ \\ 1The Laboratory for Healthcare Systems Optimization, Engineering and Informatics, Faculty of Industrial and Systems \\ Engineering, Tarbiat Modares University, Tehran, Iran \\ ${ }^{2}$ Functional Neurosurgery Research Center, Shohada Tajrish Neurosurgical Comprehensive Center of Excellence, Shahid \\ Beheshti University of Medical Sciences, Tehran, Iran \\ ${ }^{3}$ Department of Healthcare Systems Engineering, Faculty of Industrial and Systems Engineering, Tarbiat Modares University, \\ Tehran, Iran \\ ${ }^{4}$ Laser Application in Medical Sciences Research Center, Shahid Beheshti University of Medical Sciences, Tehran, Iran
}

\begin{abstract}
Background: The aim of this study was to investigate effect of some clinical attributes in prediction of satisfaction with posterior spinal fusion (PSF) surgery in patients with lumbar disc herniation (LDH) and lumbar spinal canal stenosis (LSCS) and determine a cut-off point for these attributes.

Methods: The attributes such as stenosis ratio (SR) values (described by Lurencin), Japanese Orthopaedic Association (JOA), The Zung depression scale (ZDS), duration of symptoms (in months), were investigated for 329 patients with LSCS and 151 patients with LDH separately. Patient satisfaction was recorded based on the international standard questionnaire Swiss Spinal Stenosis Score (SSS). The sensitivity and specificity values and the optimal cut-off points were calculated for SR, JOA, ZDS and duration of symptoms using receiver operating characteristic (ROC) analysis. Results: One hundred fifty-one patients with LDH (39 male, 112 female; mean age $50.24 \pm 9.21$ years) and 329 patients with LSCS (111 male, 218 female; mean age $53.28 \pm 7.81$ years) were followed-up for 6 months. Post-surgical satisfaction was $73.86 \%$ in patients with LSCS and $85.43 \%$ in patients with LDH. The cut-off point of SR for prediction of besting surgical outcome was estimated more than 0.46 with asymptotic significance less than $0.05,60 \%$ sensitivity and $75 \%$ specificity in LSCS patients (AUC-0.705, 95\% Cl, 0.644-0.766; $\mathrm{P}<0.001$ ).

Conclusion: The findings show that the SR with a cut off value of 0.46 cross sectional area, in patients with LSCS may be superior to JOA, duration of symptoms and ZDS for prediction of satisfaction with PSF surgery.

Keywords: Surgical satisfaction; Posterior spinal fusion; Stenosis ratio; Japanese Orthopaedic Association score; Zung depression scale.
\end{abstract}

*Correspondence to Alireza Zali, MD, Head of Functional Neurosurgery Research Center and Shohada Tajrish Neurosurgical

Comprehensive Center of Excellence, Shahid Beheshti

University of Medical

Sciences, Tehran, Iran.

Fax: $+98(21) 22724214$ Tel: $+98(21) 22724211$;

Email:

dr.zali.alireza@gmail.com

Published online 19 June 2018

Citation: Babaee M, Zali A, Sepehri MM, Razzaghi Z. Cut-off value for stenosis ratio and Zung depression scale in successful prediction of posterior spinal fusion surgery. Int Clin Neurosci J. 2018;5(2):46-50. doi:10.15171/icnj.2018.09.

\section{Introduction}

Low back pain is one of the most important diseases that disturb daily activities of patients. Lumbar spinal canal stenosis (LSCS) and lumbar disc herniation (LDH) are among the reasons for low back pain. LSCS refers to disease in which spinal canal becomes narrower and leads to pain, numbness and weakness in lower limbs. ${ }^{1}$ $\mathrm{LDH}$ is a shift of disc between vertebrae outside the intervertebral disc space. ${ }^{2}$ Spinal surgeries in many cases are recommended to the patients with low back pain as treatment strategy. Posterior spinal fusion (PSF) surgery is applied for treatment of LDH and LSCS when conservative treatments are not efficient. ${ }^{3}$
Analysis of diagnostic factors in order to low-cost evaluation and more precise diagnosis can assist neurosurgeons in decision making. In modern medicine, more accurate investigation of important variables, computationally, and determination of a cutoff point for them, alongside routine neuroimaging methods, can play a valuable role in low-cost and easier diagnosis of diseases in neurosurgery field.

Mohammadi et al determined the optimal cut-off point of 0.52 for SR in laminectomy surgery without fusion to specify the role of this variable in predicting surgery satisfaction in patients with LSCS, for this purpose SR was compared with Neurogenic Claudication Outcome

(C) 2018 The Author(s). This is an open access article distributed under the terms of the Creative Commons Attribution License (http:// creativecommons.org/licenses/by/4.0/), which permits unrestricted use, distribution, and reproduction in any medium, provided the original work is properly cited. 
Score (NCOC) and walking distance. ${ }^{4}$ Fujimori et al validated the JOA scoring system based on self-reported improvement and the best cutoff points and the area under the curve (AUC) for JOA were calculated for posterior lumbar interbody fusion surgery in patients with spondylolisthesis. ${ }^{5}$ Azimi et al reported an optimal cut-off point for Pain Sensitivity Questionnaire (PSQ) to achieve a more precise scale for prediction surgery outcome in patients with LDH. ${ }^{6}$ Donk et al reported that Neck Disability Index (NDI) correlated with a desirable outcome in anterior cervical surgery, for this purpose a cutoff point of seven or less was proposed for NDI. ${ }^{7}$ Matsumoto et al presented a new scoring system for preoperative decision making to diagnose malignant and benign spinal dumbbell tumors by determination of an optimum cut-off value for the dumbbell scoring system (DSS). ${ }^{8}$ The area under the ROC curve analysis as an efficient tool for prediction and classification is used in medical researchers. ${ }^{9}$

The study that compares cut-off values for effective factors in successful prediction of PSF surgery in patients with LDH and LSCS, have not been previously investigated.

The aim of this study is to specify independent and effective variables that predict outcome of PSF surgery in patients with LSCS and LDH and calculate the optimal cut-off value of these variables. In this study the optimal cut-off point of stenosis ratio (SR), Japanese Orthopaedic Association (JOA) score, duration of symptoms and the Zung depression scale (ZDS), were obtained and compared in patients with $\mathrm{LDH}$ and LSCS by receiver operating characteristic (ROC) curve analysis at the 6 months follow up.

\section{Materials and Methods \\ Patients and Data Collection}

Between January 2015 and October 2016, 151 patients with LDH and 329 patients with LSCS were referred to Shohada Tajrish Hospital as neurosurgical center of excellence of Iran, in Tehran, for PSF surgery. All the patients were treated by PSF surgery. It means only patients entered the study that PSF surgery was diagnosed as the most appropriate treatment for them by neurosurgeons. We only studied patients who were candidates for PSF surgery because of serious instability in lumbar spinal canal, this diagnosis was confirmed by at least two neurosurgeons, both in the patients with LDH and patients with LSCS. In fact, patients became candidates for PSF surgery under the condition that the "discectomy" and "decompression surgery without fusion" were not an effective treatment for them.

Imaging studies including CT and MRI of the lumbar spine and clinical examinations were investigated for all the patients with LDH and LSCS. Data for patients was included demographic such as age, gender and some of the clinical attributes of patients such as duration of symptoms (in months), JOA, The ZDS, and SR values. The patient satisfaction with surgery was assessed based on International standard questionnaire Swiss Spinal Stenosis Score (SSS). ${ }^{10}$ Postoperative assessment for patients was performed at least 6 months after surgery.

\section{Additional Measures}

The SR value is a scale to specify intensity of stenosis. This scale that is shown as a number between o and 1, is the cross-sectional area of the canal at the axial MRI image with presentation the greatest neurological compression at disc level over the cross-sectional area at the pedicle level above. ${ }^{11}$ In this work the SR is calculated for the most stenotic level.

JOA score is a tool for assessment of sensory and motor status and severity of pain in patients with low back pain. The JOA score is computed total 29 points; a high JOA score shows a better condition for patient. ${ }^{12}$

The ZDS is a 20 -item questioner that ranges between 20 and 80 . The ZDS evaluates depression in patients; the score more than 55 indicates depression. ${ }^{13}$

The SSS as an international standard questionnaire, assesses physical function characteristics, lumbar spine disability and treatment outcomes in lumbar spinal stenosis. To determine the patient satisfaction, questions 13 to 18 were evaluated, ${ }^{10}$ in this way, patients were classified as 2 classes: satisfied and unsatisfied patient.

A cut-off point for SR, the JOA score, the ZDS score and duration of symptoms were determined by ROC analysis to beget a new insight for prediction of surgical satisfaction in patients with LDH and LSCS separately.

\section{Statistical Analysis}

The ROC curve analysis and statistical calculations were performed by PASW Statistics 16 version 16 (SPSS Inc., 2007, Chicago, IL, USA) to estimate the optimal cutoff point of SR, the JOA score, the ZDS and duration of symptoms in patients with LDH and LSCS separately. Areas under the curve (AUC) were measured for comparison of the accuracy of tests and importance of variables. The high AUC as an index indicates high ability of model prediction and importance of the variable role in prediction. There is a category for different values of AUC: no discrimination (AUC $=0.50$ ), acceptable discrimination $(0.7 \leq \mathrm{AUC}<0.8)$, excellent discrimination $(0.8 \leq \mathrm{AUC}<0.9)$, and outstanding discrimination (AUC more than 0.9$).{ }^{14}$ The maximal sensitivity and specificity were calculated for each variable in patients with $\mathrm{LDH}$ and patients with LSCS separately.

\section{Results}

The demographics of the 329 patients with LSCS and their scores on the JOA, the ZDS, duration of symptoms and SR are presented in Table 1. The demographics of the 151patients with LDH and their scores on the JOA, the ZDS, duration of symptoms and SR are presented in Table 
Table 1. Clinical Attributes of Patients With LSCS Before Surgery

\begin{tabular}{ll}
\hline Characteristics & Mean \pm SD \\
\hline Age, y & $53.28 \pm 7.81$ \\
Gender (male), \% & $33.73 \%$ \\
Duration of symptoms (mon) & $115.36 \pm 75.08$ \\
Japanese Orthopaedic Association & $8.13 \pm 3.58$ \\
The Zung depression scale & $48.21 \pm 7.22$ \\
Stenosis ratio values & $0.46 \pm 0.12$ \\
\hline
\end{tabular}

Table 2. Clinical Attributes of Patients With LDH Before Surgery

\begin{tabular}{ll}
\hline Characteristics & Mean \pm SD \\
\hline Age, y & $50.24 \pm 9.21$ \\
Gender (male), \% & $25.82 \%$ \\
Duration of symptoms (mon) & $82.10 \pm 57.09$ \\
Japanese Orthopaedic Association & $7.80 \pm 3.42$ \\
The Zung depression scale & $48.78 \pm 8.95$ \\
Stenosis ratio values & $0.45 \pm 0.12$ \\
\hline
\end{tabular}

2. All the patients were treated by PSF surgery. Follow up was at least 6 months. The satisfaction with PSF surgery was $73.86 \%$ and $85.43 \%$ in patients with LSCS and LDH respectively.

Table 3 shows that the AUC, the optimal cut-off point and maximal sensitivity and specificity are calculated for $\mathrm{SR}$, the JOA score, the ZDS and duration of symptom in patients with LSCS and compared with each other. Table 3 presents that SR, ZDS and JOA score can be significant predictors for prediction of outcome surgery because of significant level less than 0.05 , although AUC values show that SR have more ability for prediction in comparison with JOA and ZDS. The AUC for the SR in Table 3 is $70.5 \%$ which is an acceptable and relatively favorable value to predict satisfaction with surgery. AUC value is determined based on besting surgical outcome and patient satisfaction so increase of SR and JOA values has a direct relationship with patient satisfaction but ZDS has an inverse relationship with patient satisfaction. It means depression in patients reduces surgical satisfaction. AUC value of ZDS for worsening surgical satisfaction is $63.7 \%$ with significant level of 0 .

Table 4 shows that the AUC, the optimal cut-off point and maximal sensitivity and specificity are calculated for $\mathrm{SR}$, the JOA score, the ZDS and duration of symptom in patients with LDH and compared with each other. Table4 presents that asymptotic significance for all the variables is more than 0.05 so none of the mentioned variables are proper predictors for prediction of satisfaction with PSF surgery in LDH patients.

According to the ROC curve analysis, the optimal cutoff point of SR was achieved 0.46 to predict besting surgical satisfaction with $60 \%$ sensitivity and $75 \%$ specificity in patients with LSCS. The finding is shown in Figure 1.

\section{Discussion}

Determination of a cut-off value for effective clinical characteristics assists clinician in decision making. The present study aimed to measure the optimal cut-off point for SR to predict success of PSF surgery in patients with LSCS and LDH in an Iranian population, and the proposed optimal cut-off point was 0.46 in patients with LSCS, using ROC analysis. It means that LSCS patients with SR $>0.46$, were more satisfied with PSF surgery and in patients with $\mathrm{LDH}$, none of the SR, JOA, ZDS and duration of symptoms, were recognized as significant predictors.

Table 3 shows that AUC values for the ZDS, duration of symptoms and the JOA score, in patients with LSCS was only $60.9 \%, 45 \%$ and $36.3 \%$ respectively, that is very lower than the SR method. It means the SR variable, in comparison with the JOA score, duration of symptoms and the ZDS, can predict satisfaction of PSF surgery more reliably. Involved spinal level was between one and six levels. Age range was between 27 and 72 in patients with LDH and between 30 and 75 in patients with LSCS.

Among patients with LSCS $11.24 \%$ and among patients with LDH $15 \%$ were smoker and the majority of patients

Table 3. Calculation of Area Under the ROC Curve and Cut-off Points for SR, JOA, ZDS and Duration of Symptoms in Patients With LSCS

\begin{tabular}{|c|c|c|c|c|c|}
\hline Model/ Parameter & AUC & $\boldsymbol{P}^{*}$ & Cut-off & Sensitivity & Specificity \\
\hline Stenosis ratio & $70.5 \%$ & 0.000 & 0.4684 & 0.601 & 0.756 \\
\hline Japanese Orthopaedic Association & $60.9 \%$ & 0.003 & 6.5 & 0.757 & 0.465 \\
\hline Duration of symptoms & $45 \%$ & 0.169 & 38.7496 & 0.852 & 0.174 \\
\hline The Zung depression Scale & $36.3 \%$ & 0.000 & 30.5 & 0.70 & 0.14 \\
\hline
\end{tabular}

Table 4. Calculation of Area Under the ROC Curve and Cut-off Points for SR, JOA, ZDS and Duration of Symptoms in Patients With LDH

\begin{tabular}{llllll}
\hline Model/ Parameter & AUC & $\boldsymbol{P}^{*}$ & Cut-off & Sensitivity & Specificity \\
\hline Stenosis ratio & $60.2 \%$ & 0.126 & 0.3428 & 0.845 & 0.409 \\
Japanese Orthopaedic Association & $54.1 \%$ & 0.539 & 10.5 & 32.5338 & 0.256 \\
Duration of symptoms & $51.5 \%$ & 0.827 & 46.0035 & 0.814 & 0.643 \\
The Zung depression Scale & $46.2 \%$ & .569 & & 0.409 \\
\hline
\end{tabular}




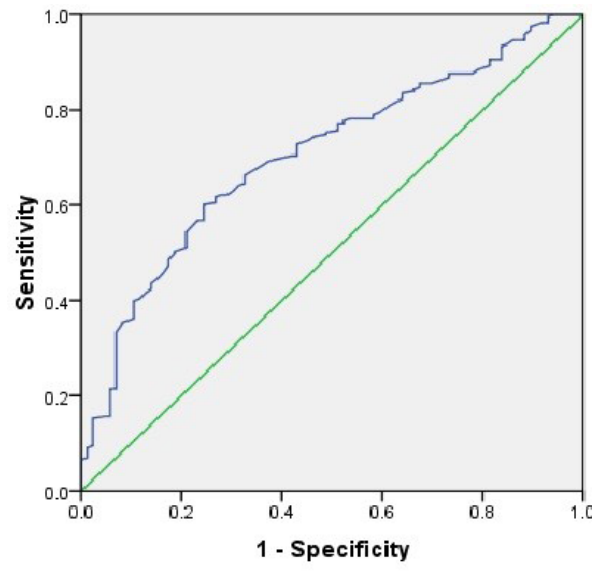

Figure 1. ROC Curve of SR for Prediction of Surgical Success in Patients With LSCS (AUC-0.70, 95\% Cl, 0.644-0.766; $P<0.001$ ).

had long duration more than one year of back and leg pain.

The SR was evaluated at the most stenotic level. There are some articles that have assessed the role of other variables in satisfaction with spinal surgery for example Sigmundsson et al performed a study in patients with central spinal stenosis without degenerative spondylolisthesis and reported that smoker and unemployed patients and patients with back pain predominance and light work were more often dissatisfied, also the patients that had duration of leg and back pain more than one year and previous surgery were more often dissatisfied. ${ }^{15}$

Sigmundsson et al performed another study to compare outcome in decompression with and without fusion surgery and showed that decompression with degenerative spondylolisthesis had better outcomes with fusion in spinal stenosis. ${ }^{16}$

Aizawa et al established a self-administered, selfreported history questionnaire as a diagnostic support tool to diagnose spinal stenosis and $\mathrm{LDH}$ by using determination of a cut-off point for each group. ${ }^{17}$

Tetreault et al determined cut-off values on the modified JOA score for mild, moderate and severe myelopathy in patients with degenerative cervical myelopathy. They reported a mJOA from 15 to 17 as mild, a mJOA from 12 to 14 as moderate and a mJOA from 0 to 11 as severe myelopathy. ${ }^{18}$

Otani et al measured a cut-off point for growth hormone levels in blood samples as an indicator and predictor of postoperation in transsphenoidal surgery. ${ }^{19}$

Takenaka et al calculated cut-off values for C-methionine, C-choline and F-fluorodeoxyglucose positron emission tomography to diagnose glioma recurrence. ${ }^{20}$

Based on the aforementioned, determination of a range or value for some important clinical factors can give an insight into the surgeon. To our knowledge, this is the first work to compare the cut-off value for SR in patients with LDH and LSCS for diagnosis of satisfaction with PSF surgery. Another report had specified SR cut-off point for prediction of surgical success (laminectomy without fusion) in patients with LSCS. ${ }^{4}$

Present study has some limitations. A limitation of this study is short-term follow-up after surgery. Further studies are needed to investigate surgical satisfaction at the more long-term follow-up. Second, because of retrospective nature of this study, there was no possibility of calculating some of the factors.

\section{Conclusion}

This work showed that the SR with a cut off value of 0.46 cross sectional area, in patients with LSCS may be preferred to ZDS, JOA and duration of symptoms to predict success in PSF surgery, but in patients with $\mathrm{LDH}$, according to the asymptotic significance more than 0.05 for SR, JOA, ZDS and duration of symptoms, none of the aforesaid variables are not acceptable for prediction of

outcome of PSF surgery.

Acknowledgements

The authors greatly appreciate Functional Neurosurgery Research Center of Shahid Beheshti University of Medical Sciences for their support.

\section{Conflict of Interest Disclosures}

The authors declare that there is no conflict of interests regarding the publication of this paper.

\section{Ethical Statement}

The Ethics Committee of Shahid-Beheshti University of Medical Sciences, Tehran, Iran, approved the study with code: IR.SBMU. RETECH.REC.1395.908.

\section{References}

1. Azimi P, Benzel EC, Shahzadi S, Azhari S, Mohammadi HR. Use of artificial neural networks to predict surgical satisfaction in patients with lumbar spinal canal stenosis: clinical article. J Neurosurg Spine. 2014;20(3):300-5. doi: 10.3171/2013.12. spine13674.

2. Azimi P, Benzel EC, Shahzadi S, Azhari S, Mohammadi HR. The prediction of successful surgery outcome in lumbar disc herniation based on artificial neural networks. J Neurosurg Sci. 2016;60(2):173-7.

3. Hayashi K, Terai H, Toyoda H, Suzuki A, Hoshino M, Tamai K, et al. Incidence of Pleural Fluid and Its Associated Risk Factors After Posterior Spinal Fusion in Patients With Adolescent Idiopathic Scoliosis. Spine (Phila Pa 1976). 2017;42(8):603-9. doi: 10.1097/brs.0000000000001861.

4. Mohammadi HR, Azimi P, Benzel EC, Shahzadi S, Azhari S. The role of stenosis ratio as a predictor of surgical satisfaction in patients with lumbar spinal canal stenosis: a receiveroperator characteristic (ROC) curve analysis. J Neurosurg Sci. 2016;60(3):345-9.

5. Fujimori T, Okuda S, Iwasaki M, Yamasaki R, Maeno T, Yamashita $T$, et al. Validity of the Japanese Orthopaedic Association scoring system based on patient-reported improvement after posterior lumbar interbody fusion. Spine J. 2016;16(6):728-36. doi: 10.1016/j.spinee.2016.01.181.

6. Azimi P, Benzel EC. Cut-Off Value for Pain Sensitivity Questionnaire in Predicting Surgical Success in Patients with Lumbar Disc Herniation. PLoS One. 2016;11(8):e0160541. doi: 10.1371/journal.pone.0160541. 
7. Donk R, Verbeek A, Verhagen W, Groenewoud H, Hosman A Bartels R. The Qualification of Outcome after Cervical Spine Surgery by Patients Compared to the Neck Disability Index. PLoS One. 2016;11(8):e0161593. doi: 10.1371/journal. pone.0161593.

8. Matsumoto $\mathrm{Y}$, Harimaya K, Kawaguchi K, Hayashida M, Okada S, Doi T, et al. Dumbbell Scoring System: A New Method for the Differential Diagnosis of Malignant and Benign Spinal Dumbbell Tumors. Spine (Phila Pa 1976). 2016;41(20):E1230-e6. doi: 10.1097/ brs.0000000000001582.

9. Fong $\mathrm{Y}$, Yin S, Huang Y. Combining biomarkers linearly and nonlinearly for classification using the area under the ROC curve. Stat Med. 2016;35(21):3792-809. doi: 10.1002/ sim.6956.

10. Azimi P, Ghandehari HS, Sadeghi S, Azhari S, Aghaei HN, Mohmmadi HR, et al. Severity of symptoms, physical functioning and satisfaction in patients with lumbar spinal stenosis: a validation study of the Iranian version of the Swiss Spinal Stenosis Score. J Neurosurg Sci. 2014;58(3):177-82.

11. Laurencin $C T$, Lipson SJ, Senatus $P$, Botchwey $E$, Jones $T R$, Koris $M$, et al. The stenosis ratio: a new tool for the diagnosis of degenerative spinal stenosis. Int J Surg Investig. 1999;1(2):127-31.

12. Azimi P, Mohammadi HR, Montazeri A. An outcome measure of functionality and pain in patients with lumbar disc herniation: a validation study of the Japanese Orthopedic Association (JOA) score. J Orthop Sci. 2012;17(4):341-5. doi: 10.1007/s00776-012-0232-x.

13. Zung WW, Richards CB, Short MJ. Self-rating depression scale in an outpatient clinic. Further validation of the SDS. Arch Gen Psychiatry. 1965;13(6):508-15.
14. Hosmer DW, Lemeshow S. Applied Logistic Regression. 2nd ed. John Wiley \& Sons, Inc; 2000:156-64.

15. Sigmundsson FG, Jonsson B, Stromqvist B. Determinants of patient satisfaction after surgery for central spinal stenosis without concomitant spondylolisthesis: a register study of 5100 patients. Eur Spine J. 2017;26(2):473-80. doi: 10.1007/ s00586-016-4495-3.

16. Sigmundsson FG, Jonsson B, Stromqvist B. Outcome of decompression with and without fusion in spinal stenosis with degenerative spondylolisthesis in relation to preoperative pain pattern: a register study of 1,624 patients. Spine J. 2015;15(4):638-46. doi: 10.1016/j.spinee.2014.11.020.

17. Aizawa T, Tanaka Y, Yokoyama T, Shimada Y, Yamazaki K, Takei $\mathrm{H}$, et al. New diagnostic support tool for patients with leg symptoms caused by lumbar spinal stenosis and lumbar intervertebral disc herniation: A self-administered, selfreported history questionnaire. J Orthop Sci. 2016;21(5):57985. doi: 10.1016/j.jos.2016.07.012.

18. Tetreault L, Kopjar B, Nouri A, Arnold P, Barbagallo G, Bartels $\mathrm{R}$, et al. The modified Japanese Orthopaedic Association scale: establishing criteria for mild, moderate and severe impairment in patients with degenerative cervical myelopathy. Eur Spine J. 2017;26(1):78-84. doi: 10.1007/s00586-016-4660-8.

19. Otani R, Fukuhara N, Ochi T, Oyama K, Yamada S. Rapid growth hormone measurement during transsphenoidal surgery: analysis of 252 acromegalic patients. Neurol Med Chir (Tokyo). 2012;52(8):558-62.

20. Takenaka S, Asano Y, Shinoda J, Nomura Y, Yonezawa S, Miwa $\mathrm{K}$, et al. Comparison of (11)C-methionine, (11)C-choline, and (18)F-fluorodeoxyglucose-PET for distinguishing glioma recurrence from radiation necrosis. Neurol Med Chir (Tokyo). 2014;54(4):280-9. 